\title{
PERMUTATIVE ZAHLEN
}

\author{
LUTZ G. LUCHT · RENATE MOTZER
}

\begin{abstract}
Zusammenfassung. Zur Untersuchung des 2017/18 von H. Hischer kommunizierten Vorkommens von 10-stelligen Dezimalzahlen, die sämtliche Ziffern $0,1, \ldots, 9$ enthalten und zu denen es einen ganzzahligen Teiler $>1$ derart gibt, dass die Division nur ihre Ziffernreihenfolge verändert, wird hier der Begriff der permutativen Zahl eingeführt. Dieser Beitrag liefert einige allgemeine Ergebnisse über Existenz und Konstruktion permutativer Zahlen und weist auf offene Probleme hin.
\end{abstract}

Revidierte Manuscript-Version vom: 19. November 2019

Erschienen in: Mathematische Semesterberichte (2020) 67: 43-55

Schlüsselworte: Verwandte Zahlen · Arithmetische Funktionen · Permutationen

2020 MSC Klassifikation: 11A25 · 05A10

\section{EINFÜHRUNG}

Ende 2017 wurde H. Hischer [1] durch die Frage seiner damals 9-jährigen Enkelin Marlene nach dem Grund für ein eigenartiges Phänomen überrascht: Nach Division der Dezimalzahl 9876543210 durch 5 enthält das Ergebnis 1975308642 sämtliche dekadischen Ziffern der Ausgangszahl wieder, nur in anderer Reihenfolge. Gibt es eine Erklärung dafür? Die Frage scheint zuvor noch nie untersucht worden zu sein, so dass eine begriffliche Präzisierung geboten erscheint. Dazu sei $\mathcal{Z}=\{0,1,2, \ldots, 9\}$ die Menge der dekadischen Ziffern und $\mathcal{Z}^{*}=\mathcal{Z} \backslash\{0\}$.

Definition 1. Es sei $a>1$ eine ganze Zahl. Existiert ein natürlicher Teiler $d>1$ von $a$ derart, dass

1. die dekadischen Darstellungen von $a$ und $m=\frac{a}{d}$ aus jeweils paarweise verschiedenen Ziffern von $\mathcal{Z}$ bestehen und

2. die Ziffernfolge von $m$ aus der von $a$ durch Permutation hervorgeht,

so nennnen wir a permutativ, genauer permutativ zum Teiler $d$, und $m$ einen permutativen Erzeuger, genauer permutativen Erzeuger zum Faktor d.

Wir beschränken die Untersuchungen vorerst auf 10-stellige dekadische Darstellungen von Zahlen, in denen jede Ziffer aus $\mathcal{Z}$ genau einmal vorkommt. Die Existenz permutativer Zahlen oder permutativer Erzeuger ist durch Marlenes Beispiel bereits geklärt:

Beispiel 1. Die Zahl $a=9876543210$ ist permutativ zu den Teilern 2,5.

Die Autoren danken Herrn Hischer für die freundliche Mitteilung der Fragestellung. 
Nachrechnen zeigt $\frac{a}{2}=4938271605$ und $\frac{a}{5}=1975308642$. Damit sind also 4938271605 und 1975308642 permutative Erzeuger zu den Faktoren 2 bzw. 5. Da 2 und 5 komplementäre Teiler von 10 sind, wird die Rechnung einfacher, wenn Division durch 5 ersetzt wird durch Multiplikation mit 2 und anschließende Division durch $m d=10$, die sich durch Weglassen der letzten Ziffer 0 erledigt. Es gibt permutative Zahlen zum Teiler 2, die nicht permutativ zum Teiler 5 sind und umgekehrt. Die folgenden Beispiele zeigen die Existenz von permutativen Zahlen zu von 2 und 5 verschiedenen Teilern.

Beispiel 2. Die Zahl $a=8641975230$ ist permutativ zu den Teilern 2, 5, 7.

Denn es gilt $\frac{a}{2}=4320987615, \frac{a}{5}=1728395046, \frac{a}{7}=1234567890$.

Zur Entdeckung permutativer Zahlen bietet sich an, sie unter den $d$-fachen geeigneter Testzahlen $t$ für Faktoren $d \in \mathcal{Z}$ mit $d>1$ zu suchen. Als Testzahlen $t$ geeignet sind dabei solche 10-stelligen Zahlen, die wieder sämtliche 10 Ziffern aus $\mathcal{Z}$ enthalten.

Beispiel 3. Zu den Faktoren $d=2,4,5,7,8$ erzeugt 1234567890 die permutativen Zahlen 2469135780, 4938271560, 6172839450, 8641975230 und 9876543120.

\section{ERZEUGER PERMUtATIVER ZAHLEN}

Die bisherigen Beispiele enthalten keine permutativen Erzeuger zu den Faktoren 3, 6, $9 \in \mathcal{Z}$. In [2] wurden durch Untersuchung von Quersummen und Überträgen bei entsprechenden Multiplikationen die Dezimalzahlen 2145973860, 1302675489 bzw. 1056384792 als zugehörige permutative Erzeuger ermittelt:

Beispiel 4. Die Zahlen 6437921580, 7816052934, 9507463128 sind permutativ zu den Teilern $d=3,6$ bzw. 9 .

Der Entdeckung liegt eine empirische Datenanalyse zugrunde, die auf folgender Idee beruht. $\mathrm{Zu} d \in \mathcal{Z}$ liefert die Menge $d \mathcal{Z}=\{d a: a \in \mathcal{Z}\}$ die $d$-fachen der Ziffern aus $\mathcal{Z}$. Sucht man permutative Erzeuger $t$ zum Faktor $d \in\{3,6,9\}$, so müssen die Ziffern des Produkts $d t$ die Menge $\mathcal{Z}$ durchlaufen.

Da 3 und 9 zu 10 teilerfremd sind, durchlaufen die Einerziffern der Zahlen aus $3 \mathcal{Z}$ und $9 \mathcal{Z}$ wieder die Elemente von $\mathcal{Z}$, haben also die Quersumme 45, und die für die Gesamtzahl der Überträge maßgebliche Summe der Zehnerziffern beträgt 9 bzw. 36. Jeder Übertrag reduziert die Summe der Ziffern der gewünschten permutativen Zahl $3 t$ um 9; daher muss es einen zusätzlichen Übertrag geben, damit $3 t$ die Quersumme 45 hat, und bei $9 t$ bedarf es vier zusätzlicher Überträge.

Zum Faktor $d=6$, der nicht zu 10 teilerfremd ist, summieren sich die Einerziffern aus $6 \mathcal{Z}$ auf 40, die Zehnerziffern auf 23. Die Summe aller Ziffern von $6 \mathcal{Z}$ ist daher 63 . Wegen $63-45=18$ sind zwei zusätzliche Überträge erforderlich.

Diese notwendigen Kriterien sind bei der Suche nach den oben angegebenen permutativen Erzeugern zu den Faktoren 3,6,9 hilfreich, reichen aber zu deren Herleitung nicht aus. 
Aussichtsreicher erscheint eine systematische Suche mit Testzahlen. Die Suchbereiche sind nach oben begrenzt, da das Ergebnis wieder 10-stellig sein muss.

Die minimale Testzahl ist offenbar 1023456789. Die maximale Testzahl $T_{d}$ für den Faktor $d$ ist durch $\frac{1}{d}\left(10^{10}-1\right)$ nach oben begrenzt, also für

$$
\begin{array}{lll}
d=3 & \text { durch } & T_{3}=3298765410 \\
d=6 & \text { durch } & T_{6}=1659874320 \\
d=9 & \text { durch } & T_{9}=1098765432 .
\end{array}
$$

Eine sämtliche möglichen Testzahlen enthaltende Tabelle, die über die Gesamtzahl aller 10-stelligen dekadisch dargestellten permutativen Zahlen Aufschluss geben und strukturelle Eigenschaften von Erzeugern permutativer Zahlen offenbaren könnte, wäre mit $21 \cdot 8$ ! = 846720 Zeilen so umfangreich, dass sie manuell nicht mehr erstellt werden kann.

\section{VARIATIONEN PERMUTATIVER ZAHLEN}

Unter Variationen permutativer Zahlen verstehen wir die systematische Generierung von permutativen Zahlen aus geeigneten Testzahlen oder aus bereits bekannten permutativen Zahlen. Zuerst analysieren wir die Ziffernpermutation, die ein permutativer Erzeuger $t$ zum Faktor 2 beim Übergang zu $a=2 t$ erfährt.

Dazu zerlegen wir die Ziffernmenge $\mathcal{Z}$ in die Menge $\mathcal{O}$ der bei Verdoppelung ohne Übertrag bleibenden Ziffern 0,1,2,3,4 und die Menge $\mathcal{M}$ der den Übertrag 1 liefernden Ziffern $5,6,7,8,9$. Indem wir die Ziffernpositonen zulässiger Erzeuger mit o bzw. * kodieren und berücksichten, das sie mit $\circ$ beginnen müssen, ergeben sich $\left(\begin{array}{l}9 \\ 4\end{array}\right)=126$ paarweise verschiedene Muster für zehnstelligen Zahlen. Da die erste Position stets mit einer Ziffer aus $\mathcal{O}^{*}=\mathcal{O} \backslash\{0\}$ beginnt, ergibt eine Abzählung $\left(\begin{array}{l}9 \\ 4\end{array}\right)=126$ zulässige Verteilungsmuster für die mit o kodierten Ziffern aus $\mathcal{O}$.

Jedes Muster gestattet $4 \cdot 4 !=96$ verschiedene zulässige Verteilungen der Elemente von $\mathcal{O}$ auf die mit $\circ$ kodierten Positionen. Danach sind die Ziffern aus $\mathcal{M}$ jedoch im allgemeinen nicht mehr frei verteilbar auf die noch verfügbaren mit $*$ kodierten Positionen: Jede bei Verdoppelung übertragsfrei bleibende Position * ist unmittelbarer Vorgänger einer Position $\circ$ oder an letzter Position; wir indizieren sie durch $\underline{*}$. Bei Verdoppelung der Ziffern aus $\mathcal{O}$ erhalten wir die geraden Ziffern 0, 2, 4, 6 oder 8, die durch etwaige Überträge auf Positionen $\circ$ ungerade werden. Die Besetzung der Positionen $*$ mit Ziffern aus $\mathcal{M}$ muss darauf an den Stellen $*$ geeignet reagieren. Die Strategie erläutern die folgenden drei Beispiele.

Beispiel 5. Tragen wir in das binäre Muster mit genau einer bei Verdoppelung übertragsfrei bleibenden Position $*$

$$
\circ \circ \circ * * * * * \underline{0} \circ
$$

eine zulässige Permutation der Ziffern aus $\mathcal{O}$ ein, etwa

$$
301 * * * * * 24,
$$


so erscheinen nach Verdoppelung bei Berücksichtigung der Überträge die Ziffern 6, 0, 3, 4, 8 . Zur Ergänzung der geraden Ziffer 2 muss an der als übertragsfrei unterstrichenen Position 6 eingetragen werden, damit die Ziffer 2 nach Verdoppelung entsteht. Die übrigen Ziffern aus $\mathcal{M}$ können nach Belieben auf die restlichen freien Positionen verteilt werden. Dafür gibt es 4! Möglichkeiten. Die dabei zum Beispiel entstehende Zahl

$$
3015789 \underline{6} 24
$$

erzeugt nun bei Verdoppelung in der Tat die permutative Zahl 6031579248. Die für dieses Muster zulässigen Permutationen der Ziffern aus $\mathcal{Z}$ liefern insgesamt $(4 \cdot 4 !) \cdot 4 !=2304$ paarweise verschiedene permutative Zahlen.

Beispiel 6. Im binären Muster

$$
\circ \underline{*} \circ \underline{*} \circ \underline{*} \circ \underline{*} \circ \underline{*}
$$

gibt es die Maximalanzahl von 5 bei Verdoppelung übertragsfrei bleibenden Positionen $*$. Bei Wahl einer zulässigen Verteilung der Ziffern aus $\mathcal{O}$, etwa

$$
1 * 0 * 2 \underline{*} 3 \underline{*} 4 \underline{*},
$$

werden sie alle durch die Überträge in ungerade Zahlen überführt. Da alle Ziffern aus $\mathcal{M}$ übertragsfrei bleiben, dürfen sie beliebig eingesetzt werden und liefern sämtliche geraden Ziffern. So erzeugt etwa

$$
1506273849
$$

bei Verdoppelung die permutative Zahl 3012547698. Insgesamt ergeben die zulässigen Ziffernpermutationen die Maximalzahl $4 \cdot 4 ! \cdot 5 !=11520$ von paarweise verschiedenen permutativen Zahlen.

Beispiel 7. Genau 3 Positionen * weist das Muster mit der binären Kodierung

$$
\circ * \underline{*} \circ \circ * \underline{*} \circ \underline{*} 0
$$

auf. Eintragung einer zulässigen Permutation der Ziffern aus $\mathcal{O}$, etwa

$$
2 * \underline{*} 34 * \underline{*} 1 * 0,
$$

ergibt nach Verdoppelung bei Beachtung der Überträge die Ziffern 5, 6, 9, 3, 0. Um darin die geraden Ziffern 2, 4,8 zu ergänzen, bedarf es der Ziffern 6, 7,9 $\in \mathcal{M}$, die an den markierten Positionen übertragsfrei nach Belieben einzufügen sind. Die restlichen Ziffern 5,8 $\in \mathcal{M}$ sind auf die zwei noch verfügbaren Positionen frei verteilbar und erzeugen bei Verdoppelung mit Übertrag die Ziffern 1 und 9. Verdoppelung der zum Beispiel entststandenen Zahl

$$
25 \underline{6} 348 \underline{7} 1 \underline{9} 0
$$

liefert die permutative Zahl 5126974380. Die zulässigen Ziffernpermutationen ergeben für dieses Muster insgesamt $(4 \cdot 4 !) \cdot(2 \cdot 3 !)=1152$ paarweise verschiedene permutative Zahlen.

Zur Bestimmung der Anzahl aller permutativen Zahlen zum Teiler 2 klassifizieren wir die Muster von potentiellen Erzeugern nach der Anzahl der von den Ziffern aus $\mathcal{M}$ bei Verdoppelung auf Positionen o wirksamen Überträge 1. Solche Positionen indizieren wir durch 
$\widehat{o}$ und erhalten für $\ell=1,2,3,4,5$ die paarweise disjunkten Musterklassen $K_{\ell}$ mit genau $\ell$ durch $\widehat{o}$ kodierten Positionen. Jede Musterklasse $K_{\ell}$ enthält neben den $\ell$ Positionen $\widehat{o}$ auch genau $\ell$ bei Verdoppelung übertragsfrei bleibende Postionen $*$. Für deren geeignete Besetzung durch Ziffern aus $\mathcal{M}$ gibt es $\ell$ ! Möglichkeiten, für die Besetzung der restlichen $5-\ell$ Positionen $*$ gibt es $(5-\ell)$ ! Möglichkeiten. Also liefert $K_{\ell}$ genau $\ell$ ! $(5-\ell)$ ! zulässige Permutationen der Elemente aus $\mathcal{M}$. Eine Abzählung der aus jedem Muster von $K_{\ell}$ resultierenden permutativen Zahlen ergibt dann $4 \cdot 4 ! \cdot \ell ! \cdot(5-\ell) !)$. Bezeichnet $\left|K_{\ell}\right|$ die Elementeanzahl der paarweise disjunkten Klasse $K_{\ell}$, so gibt es genau

$$
\begin{aligned}
& 96 \cdot \sum_{\ell=1}^{5} \ell ! \cdot(5-\ell) ! \cdot\left|K_{\ell}\right| \\
& \quad=96 \cdot\left(120 \cdot\left|K_{5}\right|+24 \cdot\left|K_{1} \cup K_{4}\right|+12 \cdot\left|K_{2} \cup K_{3}\right|\right)
\end{aligned}
$$

permutative Zahlen zum Teiler 2. Das folgende Lemma informiert über die benötigten Elementeanzahl der disjunkten Musterklassen.

Lemma 1. Es gilt $\left|K_{4}\right|=20,\left|K_{1}\right|=5,\left|K_{5}\right|=1$ sowie $\left|K_{2} \cup K_{3}\right|=100$.

Zum Beweis bestimmen wir zuerst die Muster mit 4 Überträgen. Da alle Muster mit einer der durch $\circ$ kodierten Ziffer aus $\mathcal{O}^{*}$ beginnen, haben 4 Überträge zur Folge, dass es keine drei aufeinanderfolgenden gleich kodierten Positionen $\circ \circ \circ$ oder $* * *$ geben kann. Daher kommt genau ein Paar o o oder genau ein Paar ** vor. Wir ordnen die 20 Muster nach der jeweils frühest möglichen Position * :

$$
\begin{aligned}
& \widehat{o} * * \widehat{o} * \widehat{o} * \widehat{o} * 0 \quad \widehat{o} * \widehat{o} * \widehat{o} * 0 \widehat{o} * * \\
& \widehat{o} * * \widehat{o} * \widehat{o} * \circ \widehat{o} * \quad \widehat{o} * \widehat{o} * \circ \widehat{o} * * \widehat{o} * \\
& \widehat{o} * * \widehat{o} * \widehat{\circ} \widehat{o} * \widehat{o} * \quad \widehat{o} * \widehat{o} * \circ \widehat{o} * \widehat{o} * * \\
& \widehat{o} * * \widehat{\circ} \widehat{o} * \widehat{o} * \widehat{o} * \quad \widehat{o} * \circ \widehat{o} * \widehat{o} * \widehat{o} * \\
& \widehat{o} * \widehat{o} * * \widehat{o} * \widehat{o} * 0 \quad \widehat{o} * 0 \widehat{o} * \widehat{o} * * \widehat{o} * \\
& \widehat{o} * \widehat{o} * * \widehat{o} * \widehat{\circ} \widehat{o} * \quad \widehat{o} * \circ \widehat{o} * \widehat{o} * \widehat{o} * * \\
& \widehat{o} * \widehat{o} * * \widehat{\circ} \widehat{o} * \widehat{o} * \quad \circ \widehat{o} * * \widehat{o} * \widehat{o} * \widehat{o} * \\
& \widehat{o} * \widehat{o} * \widehat{o} * * \widehat{o} * 0 \quad 0 \widehat{o} * \widehat{o} * * \widehat{o} * \widehat{o} * \\
& \widehat{o} * \widehat{o} * \widehat{o} * * \widehat{\circ} \widehat{o} * \quad \widehat{o} * \widehat{o} * \widehat{o} * * \widehat{o} * \\
& \widehat{o} * \widehat{o} * \widehat{o} * \widehat{o} * * 0 \quad 0 \widehat{o} * \widehat{o} * \widehat{o} * \widehat{o} * * \text {. }
\end{aligned}
$$

Jedes Muster mit nur einem Übertrag auf durch o kodierten Ziffern aus $\mathcal{O}^{*}$ enthält notwendig die komplette Musterkette $* * * * *$ für die Ziffern aus $\mathcal{M}$. Sie ist in genau 5 Mustern 
enthalten:

$$
\begin{aligned}
& \circ \circ \circ \circ \widehat{\circ} * * * * * \\
& \circ \circ \circ \widehat{\circ} * * * * * \circ \\
& \circ \circ \widehat{\circ} * * * * * \circ \circ \\
& \circ \widehat{\circ} * * * * * \circ \circ \circ \\
& \widehat{\circ} * * * * * \circ \circ \circ \circ .
\end{aligned}
$$

Aus der Gesamtzahl 126 aller paarweise verschiedenen Muster und der bisher bestimmten Werte $K_{\ell}$ für $\ell=1,4,5$ kommt $K_{2} \cup K_{3}=126-1-20-5=100$, wie behauptet.

Einsetzen der Werte aus Lemma 1 in (1) liefert

$$
96 \cdot(120+600+1200)=184320
$$

permutative Zahlen. Sie kommen daher ziemlich oft vor:

Satz 1. Es gibt 184320 permutative Zahlen zum Teiler 2 mit 10-stelliger dekadischer Darstellung.

Eine entsprechende Konstruktion aller permutativen Zahlen ist nur noch zu dem zu 2 komplementären Teiler 5 von 10 möglich. Wir erhalten sie aus Erzeugern zum Faktor 2, die nach Verdoppelung 11-stellig und nach ganzzahliger Division durch 10 permutativ sind. Zulässige Erzeuger müssen offenbar mit einer Ziffer aus $\mathcal{M}$ beginnen und mit einer der beiden Ziffern 0 oder 5 enden.

Aus den 10-stelligen Anordnungen mit $*$ an erster und 0 bzw. 5 an letzter Position, wobei der Unterstrich den bei Verdoppelung entstehenden Übertrag auf die davor stehende Position indiziert, bekommen wir $\left(\begin{array}{l}8 \\ 4\end{array}\right)=70$ bzw. $\left(\begin{array}{l}8 \\ 5\end{array}\right)=42$ zulässige paarweise verschiedene Muster. Die Anzahl der zulässigen Verteilungen der Ziffern aus $\mathcal{O}^{*}$ beträgt 4! bzw. 4 • 4!. Die zulässigen Verteilungen der Ziffern aus $\mathcal{M}$ bzw. $\mathcal{M} \backslash\{5\}$ hängt wieder von den Positionen der Ziffern aus $\mathcal{O}$ ab.

Klassifizierung der Muster nach der Anzahl der Positionen $\widehat{o}$, wobei $\widehat{o}$ wieder den bei Verdoppelung wirksamen Übertrag 1 auf die davorstehende Position anzeigt, bestimmt schließlich die Anzahl der permutativen Zahlen zum Teiler 5. Sie ist geringer als die der permutativen Zahlen zum Teiler 2, aber ähnlich aufwändig zu erhalten. Zudem ist zu beachten, dass darunter permutative Zahlen sind, die zugleich permutativ zum Teiler 2 sind, und dass es darüber hinaus permutative Zahlen zu von 2 und 5 verschiedenen Teilern gibt.

\section{4. $g$-PERMUTATIVE ZAHLEN}

Ist $g \geq 2$ eine feste natürliche Zahl, so lässt sich als Folge des Euklidischen Algorithmus, also der ganzzahligen Division mit (kleinstem nichtnegativen) Rest, jede positive ganze Zahl $a$ auch $g$-adisch darstellen, worunter eine Darstellung der Form

$$
a=a_{\ell-1} \cdot g^{\ell-1}+a_{\ell-2} \cdot g^{\ell-2}+\cdots+a_{2} \cdot g^{2}+a_{1} \cdot g+a_{0}
$$


verstanden wird. Als Koeffizienten $a_{\ell-1}, a_{\ell-2}, \ldots, a_{2}, a_{1}, a_{0}$ mit $a_{\ell-1} \neq 0$ dienen die Ziffern $a_{\lambda}$ aus der Menge

$$
\mathcal{Z}_{g}=\{0,1,2, \ldots, g-2, g-1\} .
$$

Die natürliche Zahl $\ell$ heißt die Länge oder Stellenzahl der obigen Darstellung von $a$. Als $g$-adische Darstellung der Null wird $0=0 \cdot g^{0}$ erklärt, ihr wird keine Länge zugeordnet. Bei fester Basis $g$ sind die Koeffizienten und damit die $g$-adische Darstellung von $a$ stets eindeutig bestimmt. Etwa hat die Eins die einstellige $g$-adische Darstellung $1=1 \cdot g^{0}$. Neben der dezimalen Darstellung mit $\mathcal{Z}_{10}=\mathcal{Z}$ sind etwa auch binäre, oktale, duodezimale, hexadezimale und sexagesimale Darstellungen zu den Basen $g=2, g=8, g=12, g=16$ bzw. $g=60$ gebräuchlich.

Als Kurzform der obigen $g$-adisch dargestellten Zahl $a$ verwenden wir

$$
a=a_{\ell-1} a_{\ell-2} \ldots a_{2} a_{1} a_{0 g},
$$

wobei der Index $g$ daran erinnert, dass die Ziffer $a_{\lambda}$ die Vielfachheit von $g^{\lambda}$ angibt.

Ersetzen wir in der Definition der permutativen Zahl die Ziffernmenge $\mathcal{Z}$ durch $\mathcal{Z}_{g}$ mit einer natürlichen Basis $g \geq 2$, so bekommen wir den Begriff der g-permutativen Zahl. Wir betrachten nur $g$-permutative Zahlen mit $g$-stelliger $g$-adischer Darstellung und fragen nach deren Existenz. Dabei unterscheiden wir den Fall von Primzahlen $g$ von dem zusammengesetzter Zahlen $g=d m$ mit $1<d<m$ und $1<m<g$. Im letzten Fall können wir Beispiel 1 modifizieren und erhalten

Satz 2. Es sei $g=d m \geq 4$ mit $1<d<g$ und $1<m<g$ eine zusammengesetzte Zahl. Dann gibt es g-permutative Zahlen mit g-stelliger g-adischer Darstellung.

Der Nachweis verwendet einige Notationen und Regeln aus der elementaren Vektorrechnung. Vektoren $\boldsymbol{v}=\left(v_{1}, \ldots, v_{m}\right)$ sind hier Folgen von $m$ ganzen Zahlen, die den Rechenregeln $c \boldsymbol{v}=\left(c v_{1}, \ldots, c v_{m}\right)$ und $\boldsymbol{v}+\boldsymbol{w}=\left(v_{1}+w_{1}, \ldots, v_{m}+w_{m}\right)$ für ganze Zahlen $c$ und Vektoren $\boldsymbol{v}, \boldsymbol{w}$ genügen. Das innere Produkt von zwei derartigen Vektoren $\boldsymbol{v}, \boldsymbol{w}$ ist die ganze Zahl $\boldsymbol{v} \cdot \boldsymbol{w}=v_{1} w_{1}+\cdots+v_{m} w_{m}$.

Die $g$-adische Darstellung der positiven ganzen Zahl

$$
a=(g-1) g^{g-1}+(g-2) g^{g-2}+\cdots+2 g^{2}+1 g^{1}+0 g^{0},
$$

lässt sich als inneres Produkt des Vektors $\boldsymbol{s}=(g-1, g-2, \ldots, 2,1,0)$ ihrer Koeffizienten und des Vektors $\boldsymbol{b}=\left(g^{g-1}, g^{g-2}, \ldots, g^{2}, g^{1}, g^{0}\right)$ von $g$-Potenzen interpretieren,

$$
a=s \cdot b .
$$

Division von $a$ durch $m$ ist gleichwertig zur Multiplikation von $a$ mit $d$ und anschließender Division von $d a$ durch $g$. Das vereinfacht die Strukturanalyse des Übergangs von der $g$ adischen Darstellung von $a$ zu der von $\frac{a}{m}$.

Zum Beweis des Satzes unterteilen wir die Vektoren $s$ und $\boldsymbol{b}$ in jeweils $d$ Teilvektoren

$$
\begin{aligned}
& \boldsymbol{s}_{k}=(k m+m-1, \ldots, k m+1, k m), \\
& \boldsymbol{b}_{k}=\left(10^{k m+m-1}, \ldots, 10^{k m+1}, 10^{k m}\right)
\end{aligned}
$$


für $k=d-1, d-2, \ldots, 2,1,0$. Dann ist $a=\boldsymbol{s}_{d-1} \cdot \boldsymbol{b}_{d-1}+\cdots+\boldsymbol{s}_{0} \cdot \boldsymbol{b}_{0}$. Offenbar ergeben sich $\boldsymbol{s}$ und $\boldsymbol{b}$ durch Aneinanderreihung der Segmente $\boldsymbol{s}_{d-1}, \boldsymbol{s}_{d-2}, \ldots, \boldsymbol{s}_{1}, \boldsymbol{s}_{0}$ bzw. $\boldsymbol{b}_{d-1}, \boldsymbol{b}_{d-2}, \ldots, \boldsymbol{b}_{1}, \boldsymbol{b}_{0}$. Multiplikation des Vektors $\boldsymbol{s}_{k}$ mit $d$ liefert wegen $d m=g$ den entsprechenden Teilvektor

$$
\begin{aligned}
d \boldsymbol{s}_{k} & =(k g+d(m-1), \ldots, k g+d, k g) \\
& =k g(1, \ldots, 1,1)+d(m-1, \ldots, 1,0)=k g \mathbf{1}+d \boldsymbol{s}_{0}
\end{aligned}
$$

von $d \boldsymbol{s}$. Dabei bezeichnet 1 den Vektor $(1, \ldots, 1)$ mit $m$ Komponenten 1.

Daraus sehen wir, dass $d \boldsymbol{s}_{0}$ die durch $d$ teilbaren Zahlen zwischen 0 und $m-1$ durchläuft, während $k g \mathbf{1}$ den Übertrag $k$ auf die jeweils davorstehende Position der $g$-Potenzen aus $\boldsymbol{b}_{k}$ sowie die letzte Position von $\boldsymbol{b}_{k+1}$ liefert. Also durchläuft $d \boldsymbol{s}_{k}$ sämtliche mit Rest $k$ durch $d$ teilbaren Zahlen zwischen $k g$ und $k g+d(m-1)$. Für $k=0,1, \ldots, d-1$ durchlaufen folglich die in $d \boldsymbol{s}$ auftretenden Ziffern sämtliche Reste modulo $g$, und zwar genau einmal mit Ausnahme der Ziffer 0. Diese tritt genau zweimal auf, nämlich jeweils an der letzten Position von $d \boldsymbol{s}_{0}$ und $d \boldsymbol{s}_{1}=g \mathbf{1}+d \boldsymbol{s}_{0}$. Bei Division von $d \boldsymbol{s}$ durch $g$ fällt die an letzter Position in $d \boldsymbol{s}_{0}$ stehenden Ziffer 0 weg, und die Ziffer $g-1$ rückt als Übertrag von $d \boldsymbol{s}_{g-1}$ an die erste Position. Also ist die Zahl $a$ aus (2) $g$-permutativ.

Dem Beweis von Satz 2 entnehmen wir, dass durch Permutation der jeweils ersten $m-1$ Ziffern innerhalb der $d$ Segmente $\boldsymbol{s}_{k}$ für $k=0,1, \ldots, d-1$, wobei jeweils die Ziffern $k m$ fixiert bleiben, insgesamt sogar $((m-1) !)^{d} g$-permutative Zahlen entstehen. Dies notieren wir als

Bemerkung 1. Zu zusammengesetzten Zahlen $g=d m \geq 4$ mit $1<d<g$ und $1<m<g$ gibt es mindestens $((m-1) !)^{d}$ g-permutative Zahlen mit g-stelliger g-adischer Darstellung.

Da die Faktoren in $g=m d$ nicht teilerfremd sein müssen, folgt neben $f(9) \geq 8$ auch die explizite 9-adische Darstellung der entsprechenden 9-permutativen Zahlen aus Bemerkung 1.

Beispiel 8. Die Zahl $a=876543210_{9}=3 \cdot 285174063_{9}$ ist 9-permutativ, ebenso die Zahlen $876543120_{9}, 876453210_{9}, 876453120_{9}, 786543210_{9}, 786543120_{9}, 786453210_{9}, 786453120_{9}$.

Die manuelle Durchführung der Multiplikation ist für $g \neq 10$ ungewohnt. Es bietet sich, die Multiplikation der Ziffern zu separieren, jeweils zunächst dekadisch durchzuführen, im nächsten Schritt jeweils $g$-adisch darzustellen (Division durch $g$ mit Rest) und anschließend mit Hilfe etwaiger Überträge wieder $g$-adisch zusammenzufassen. Im ersten Fall von Beispiel 6 sieht das ausführlich etwa so aus:

$$
\begin{aligned}
3 \cdot 285174063_{9} & =|06| 24|15| 03|21| 12|00| 18|09|_{10} \\
& =|06| 26|16| 03|23| 13|00| 20|10|_{9} \\
& =876543210_{9} .
\end{aligned}
$$

Erzeuger $t$ von $g$-permutativen Zahlen $m$ zu Faktoren $d \in \mathcal{Z} \backslash\{0,1\}$ müssen der generellen Bedingung genügen, dass ihre $g$-adische Darstellung $g$-stellig ist und jede Ziffer aus $\mathcal{Z}_{g}$ 
enthält. Ist $m=d t$ tatsächlich permutativ, so stimmt die Quersumme $Q_{g}(d)$ der $g$-adisch dargestellten Elemente aus $d \mathcal{Z}_{g}$ überein mit der von $\mathcal{Z}_{g}$, also

$$
Q_{g}(1)=\sum_{k \in \mathcal{Z}_{g}} k=\frac{1}{2}(g-1) g .
$$

Da jeder einzelne Übertrag bei der Berechnung von $Q_{g}(m)$ in der $g$-adischen Darstellung der Elemente von $d \mathcal{Z}_{g}$ die $d$-fache Summe $d Q_{g}(1)$ um $g-1$ reduziert, braucht es dazu

$$
(d-1) S_{g} \cdot \frac{1}{g-1}=\frac{(d-1) g}{2}
$$

Überträge. Dies erfordert die ganzzahlige Teilbarkeit von $(d-1) g$ durch 2 und reduziert für ungerade Basen $g$ die Anzahl der zu überprüfenden Muster beträchtlich:

Folgerung 1. Ist $g \geq 3$ ungerade, so existieren keine g-permutativen Erzeuger zu geraden Faktoren $d \in \mathcal{Z}_{g} \backslash\{0,1\}$.

Bemerkung 2. Die Variationsmethode aus Abschnitt 3 lässt sich auf zusammengesetzte Basen $g=d m$ mit $d=2$ ausdehnen, weil sich die Ziffernmenge $\mathcal{Z}_{g}$ in die gleichmächtigen disjunkten Teilmengen $\mathcal{O}=\{0,1,2, \ldots, m-1\}$ und $\mathcal{M}=\{m+1, m+2, \ldots, 2 m-1\}$ zerlegen lässt, wobei die bei Verdoppelung aus $\mathcal{O}$ entstehenden g-adischen Zahlen ohne Übertrag bleiben, wärend die aus $\mathcal{M}$ mit Übertrag 1 zweistellig werden. Die Anzahl der durch Variation erhaltenen g-permutativen Zahlen zum Teiler 2 ist im allgemeinen wesentlich höher als die aus der zum Beweis von Satz 1 entwickelte Konstruktionsmethode kommende Anzahl.

\section{EXKURS ZU $g$-PERMUTATIVEN ZAHLEN FÜR $g \leq 10$}

Es bezeichne $\mathbb{N}$ die Menge der positiven ganzen Zahlen, $f(g)$ die Anzahl der $g$-stelligen $g$-permutativen Zahlen und $f: \mathbb{N} \rightarrow \mathbb{N} \cup\{0\}$ die zugehörige Zählfunktion. Für $g \leq 10$ lassen sich die Werte $f(g)$ aus den bisherigen Ergebnissen bestimmen oder der Umfang der Klassen $K_{g}=\{a \in \mathbb{N}: a g$-permutativ $\}$,nichttrivial“" nach unten abschätzen.

Folgerung 2. Es gelten die nachstehenden Aussagen:
a. $f(1)=f(2)=f(3)=0$.
b. $f(4)=5, K_{4}=\left\{2130_{4}, 3012_{4}, 3210_{4}, 3120_{4}, 3201_{4}\right\}$
c. $f(5)=1, K_{5}=\left\{43120_{5}\right\}$.
d. $f(6)=138$.
e. $f(7) \geq 1$.
f. $f(8) \geq 3456$ (Anzahl der 8-permutativen Zahlen zum Teiler 2).
g. $f(9) \geq 8$.
h. $f(10) \geq 184320$ (Anzahl der 10-permutativen Zahlen zum Teiler 2).

Der Nachweis beruht auf unterschiedlichen Argumenten. 
a. Die Definition $g$-permutativer Zahlen hat zur Folge $f(1)=f(2)=0$. Als einzige Testzahl für $g=3$ mit der Ziffernmenge $\mathcal{Z}_{3}=\{0,1,2\}$ kommt nur $102_{3}$ in Frage. Verdoppelung führt zur nicht permutativen Zahl $211_{3}$. Daher gilt $f(3)=0$.

b. Für $g=4$ ist $\mathcal{Z}_{4}=\{0,1,2,3\}$. Als mögliche 4-permutative Erzeuger zum Faktor 2 kommen nur Zahlen in Frage, deren 4-adische Entwicklung mit der Ziffer 1 beginnt: 1032 $1203_{4}, 1230_{4}, 1302_{4}$ sowie $1320_{4}$. Verdoppelung zeigt, dass die vier Produkte $2 \cdot 1032_{4}=$ $2130_{4}, 2 \cdot 1203_{4}=3012_{4}, 2 \cdot 1230_{4}=3120_{4}$ sowie $2 \cdot 1302_{4}=3210_{4}$ 4-permutativ sind. Mögliche 4-permutative Erzeuger zum Faktor 3 sind die geraden Zahlen, deren 4-adische Entwicklung mit der Ziffernfolge 10 beginnt, also $1023_{4}$ und $1032_{4}$. Verdreifachung zeigt, dass nur das Produkt $3 \cdot 1023_{4}=3201_{4}$ 4-permutativ ist. Insgesamt gibt es daher genau die fünf in $K_{4}$ angegebenen 4-permutativen Zahlen.

c. Für $g=5$ ist $\mathcal{Z}_{5}=\{0,1,2,3,4\}$. Als Faktoren für zulässige Erzeuger $t$ kommt nach Folgerung 1 nur $d=3$ in Frage. Daher gilt $t \leq \frac{1}{3} \cdot\left(5^{5}-1\right)$, also $t \leq M_{3}=13042_{5}$. Wir klassifizieren nach den möglichen Mustern mit ein oder zwei Paaren $\circ *$ von aufeinanderfolgenden Ziffern, wobei die bei Verdreifachung ohne Übertrag bleibenden Ziffern aus $\mathcal{O}=\{0,1,2\}$ durch $\circ$, die den Übertrag 1 oder 2 liefernden Ziffern aus $\mathcal{M}=\{3,4\}$ durch * kodiert und Überträge auf die Ziffern aus $\mathcal{O}$ durch $\widehat{o}$ angezeigt werden:

$$
\begin{array}{ll}
\widehat{o} * * \circ \circ & \circ \widehat{o} * * \circ \\
\widehat{o} * \widehat{o} * \circ & \circ \widehat{o} * \widehat{o} * \\
\widehat{\circ} * \circ \widehat{o} * & \circ \circ \widehat{o} * *
\end{array}
$$

Da die erste Position stets mit 1 besetzt ist, gibt es für jedes Muster maximal 4, insgesamt also maximal 24 Testzahlen zum Faktor 3. Als einziges Muster liefert $\circ \widehat{o} * * \circ$ eine 5permutative Zahl, nämlich $43120_{5}=3 \cdot 12340_{5}$.

d. Für $g=6$ mit $\mathcal{Z}_{6}=\{0,1,2,3,4,5\}$ sei $\mathcal{O}=\{0,1,2\}$ und $\mathcal{M}=\{3,4,5\}$. Die Musterkonstruktion für permutative Zahlen zum Teiler 2 lässt sich wie die in Abschnitt 3 mit $g=10$ durchführen: Da zulässige Muster mit einer Ziffer aus $\mathcal{O}^{*}$ beginnen, gibt es genau $\left(\begin{array}{l}5 \\ 2\end{array}\right)=10$ solche Muster mit je drei durch $\circ$ und $*$ kodierten Positionen für die Ziffern aus $\mathcal{O}$ und $\mathcal{M}$. Klassifikation der Muster nach der Anzahl der bei Verdoppelung mit dem Übertrag 1 bedachten Positionen o, beschrieben durch $\widehat{o}$, ergibt jeweils $2 \cdot 2 !=4$ zulässige Verteilungen der Ziffern aus $\mathcal{O}$. Die zulässigen Verteilungen der Ziffern aus $\mathcal{M}$ ist abhängig von der Anzahl der auftretenden Positionen $\widehat{o}$. Auf die Klassen $K_{\ell}$ für $\ell=1,2,3$ entfallen 3, 6 und 1 Muster mit 2, 2 und 6 zulässigen Verteilungen der Ziffern aus $\mathcal{M}$. Die Anzahl der 6-permutativen Zahlen zum Teiler 2 ist daher $4 \cdot(3 \cdot 2+6 \cdot 2+1 \cdot 6)=96$.

Systematisches Ausprobieren mit Hilfe eines Tabellenkalkulationsprogramms ergibt als Anzahl 6-permutativer Zahlen zum Teiler 3 noch 48, wobei 9 zugleich 6-permutativ zum Teiler 2 sind, zum Teiler 5 genau 1 neue, $531024_{6}=5 \cdot 103452_{6}$, sowie zum Teiler 4 weitere 5, die jedoch schon 6-permutativ zum Teiler 2 sind. Insgesamt kommt daraus $f(6)=96+(48-9)+1=138$. 
e. Für $g=7$ ist $\mathcal{Z}_{7}=\{0,1,2,3,4,5,6\}$. Faktoren für 7-adische permutative Erzeuger können nach Folgerung 1 nur 3 und 5 sein. Experimentelles Testen liefert die 7-permutative Zahl $3104625_{7}=3 \cdot 1023654_{7}$ zum Faktor 3, wie leichte Rechnung bestätigt.

f. Im Fall $g=8$ mit $\mathcal{Z}_{8}=\{0,1,2,3,4,5,6,7\}$ sei $\mathcal{O}=\{0,1,2,3\}$ und $\mathcal{M}=\{4,5,6,7\}$. Die Musterkonstruktion für permutative Zahlen zum Teiler 2 lässt sich wie die aus Abschnitt 3 für $g=10$ durchführen: Da zulässige Muster mit einer Ziffer aus $\mathcal{O}^{*}$ beginnen, gibt es genau $\left(\begin{array}{l}7 \\ 3\end{array}\right)=35$ solche Muster mit je vier durch $\circ$ und $*$ kodierten Positionen für die Ziffern aus $\mathcal{O}$ und $\mathcal{M}$. Klassifikation der Muster nach der Anzahl der bei Verdoppelung mit dem Übertrag 1 bedachten Positionen $\circ$, beschrieben durch $\widehat{o}$, ergibt jeweils $3 \cdot 3 !=18$ zulässige Verteilungen der Ziffern aus $\mathcal{O}$. Die zulässigen Verteilungen der Ziffern aus $\mathcal{M}$ ist abhängig von der Anzahl der auftretenden Positionen $\widehat{o}$. Auf die Klassen $K_{\ell}$ für $\ell=1,2,3,4$ entfallen 4, 18, 12 und 1 Muster mit 6, 4, 6 und 24 zulässigen Verteilungen der Ziffern aus $\mathcal{M}$. Als genaue Anzahl der 8-permutativen Zahlen zum Teiler 2 kommt daraus $18 \cdot(4 \cdot 6+18 \cdot 4+12 \cdot 6+1 \cdot 24)=3456$.

g. Für $g=9$ ist $\mathcal{Z}_{9}=\{0,1,2,3,4,5,6,7,8\}$, und als Faktoren zu 9-adischen Erzeugern eignen sich nur 3, 5 und 7. Insbesondere entnehmen wir der Bemerkung 1, dass es für $d=$ $m=3$ mindestens $2^{3}=8$ permutative Zahlen gibt, nämlich entsprechend der Konstruktion gemäß Satz 2 die in Beispiel 8 angegebenen.

h. Der Nachweis ist durch Satz 1 erledigt.

Damit ist die Frage nach der Existenz von $g$-permutativen Zahlen für alle natürlichen Zahlen $g$ mit Ausnahme der Primzahlen $g \geq 10$ geklärt.

\section{Offene Fragen}

Wie bei vielen einfach zu formulierenden und neuartig erscheinenden Fragen stehen am Anfang experimentelle Entdeckungen, wie sie hier beschrieben sind. Es bleibt die Aufgabe, neue Ansätze für eine mathematisch befriedigende Erklärung der permutativen Zahlen zu gewinnen:

1. Wie groß ist die Anzahl $f(10)$ der permutativen Zahlen mit 10-stelliger Dezimaldarstellung?

2. $\mathrm{Zu}$ welchen Primzahlen $g \geq 11$ gibt es $g$-permutative Zahlen mit $g$-stelliger $g$ adischer Zahldarstellung?

3. Welche Aussagen lassen sich über die arithmetische Funktion $f$ und ihre Struktur machen?

\section{LITERATUR}

[1] H. Hischer, Marlene und den Zahlen: Permutationen durch Variationen, GDM-Mitteilungen 105, 29-30 (2018).

[2] R. Motzer, Entdeckungen zu Marlene und den Zahlen: Permutationen durch Variationen, GDMMitteilungen 106, 6-7 (2019). 
Lutz G. Lucht · Siemensstr. 1 - 38640 Goslar

Email address: m.lg@online.de

Renate Motzer · Universitätsstr. $10 \cdot 86135$ Augsburg

Email address: renate.motzer@math.uni-augsburg.de 